\title{
LACTB suppresses carcinogenesis in lung cancer and regulates the EMT pathway
}

\author{
YIHUI XU ${ }^{1}$, HUBO SHI $^{2}$, MIN WANG $^{1}$, PING HUANG ${ }^{1}$, MINGJIE XU $^{1}$, \\ SHUYI HAN ${ }^{1}$, HUANJIE $\mathrm{LI}^{3}$ and YUNSHAN WANG ${ }^{1,3}$
}

\begin{abstract}
${ }^{1}$ Medical Research and Laboratory Diagnostic Center, Central Hospital Affiliated to Shandong First Medical University, Jinan, Shandong 250013; ${ }^{2}$ Department of Thoracic Surgery, Shangdong Public Health Clinical Center, Jinan, Shandong 250102;

${ }^{3}$ School of Medicine, Cheeloo College of Medicine, Shandong University, Jinan, Shandong 250012, P.R. China
\end{abstract}

Received October 26, 2021; Accepted December 31, 2021

DOI: $10.3892 /$ etm.2022.11172

\begin{abstract}
Lung cancer causes thousands of deaths worldwide every year, and present therapeutics show little benefit for advanced-stage patients. Researchers do not know why and how lung cancer begins. Lactamase $\beta(L A C T B)$ is a tumor-suppressor in some cancers. However, its role in lung cancer is unknown. By analyzing the TCGA database and Kaplan-Meier Plotter database, LACTB was found to be downregulated in lung cancer tissues but the methylation level was increased. Patients with high $L A C T B$ expression exhibited improved survival. Then, in vitro assays demonstrated that $L A C T B$ overexpression inhibited cell migration and invasion, and induced apoptosis in H1299 and H1975 cells. Knockdown of $L A C T B$ caused the reverse effects. Moreover, a much higher apoptotic rate and more potent inhibitory effects on H1299 and H1975 cells were obtained when LACTB was combined with docetaxel. In addition, members of the epithelial-mesenchymal transition (EMT) signaling pathway were assessed using western blot analysis. The expression of E-cadherin was decreased while levels of $\mathrm{N}$-cadherin and vimentin were increased after knockdown of $L A C T B$ in lung cancer cells. By contrast, overexpression of $L A C T B$ increased the level of E-cadherin but decreased $\mathrm{N}$-cadherin and vimentin. Therefore, $L A C T B$ is a tumor suppressor in lung cancer that inhibits cell migration and invasion and induces cell apoptosis. Meanwhile, $L A C T B$ was found to strengthen the anticancer
\end{abstract}

Correspondence to: Professor Yunshan Wang, Medical Research and Laboratory Diagnostic Center, Central Hospital Affiliated to Shandong First Medical University, 105 Liberate Road, Jinan, Shandong 250013, P.R. China

E-mail: jnwyszxyy@163.com

Dr Huanjie Li, School of Medicine, Cheeloo College of Medicine, Shandong University, 44 West Wenhua Road, Jinan, Shandong 250012, P.R. China

E-mail: lihuanjie@sdu.edu.cn

Key words: LACTB, lung cancer, epithelial-mesenchymal transition, docetaxel role of docetaxel and to suppress the EMT pathway in lung cancer.

\section{Introduction}

Lung cancer is the most lethal malignancy in the world and causes approximately 132 thousand deaths each year. A total of 235,700 patients are diagnosed each year according to the newest cancer statistical report by the American Cancer Society (ACS) (1). In China, the data were astonishing due to the large population. The average incidence was 733,300 and the number of deaths was 610,200 (2). At present, the main strategy for lung cancer is still surgical resection followed by drug chemotherapy and/or radiation therapy (3). However, the majority of patients do not benefit from traditional therapy. Approximately $57 \%$ of the patients diagnosed with lung cancer have distant metastasis while $17 \%$ have a localized distribution (1). The patient's survival is dependent on their cancer stage. The five-year survival rate of patients with distant metastasis is only 6\% (1). There is little time left for us to identify more potent weapons to fight lung cancer.

Lung cancer tends to metastasize due to its high genetic heterogeneity (4). From the emergence of second-generation sequencing technology, a list of genetic factors including mRNAs, long non-coding (lnc)RNAs, and microRNAs (miRNAs) have been detected that take part in the progression or metastasis of lung cancer. For example, miR-196b was reported to inhibit cell metastasis of lung cancer (5). Loss of E-cadherin was found to promote cell metastasis in lung cancer (6). However, in a cohort of lung cancer patients, the dominant genetic factors are always different, which suggests that it is a long journey to determine the deep underlying mechanisms of lung cancer.

Lactamase $\beta(L A C T B)$ is a gene mapped to chromosome $15 q 22.2$ that encodes a mitochondrially localized $\beta$-lactamase. It is reported to play suppressor roles in the metastasis and progression of various type of cancer. For example, LACTB was found to suppress the migration and invasion of melanoma and to block the lung metastasis of melanoma cells (7). In glioma, LACTB inhibited the proliferation, invasion and angiogenesis of tumor cells (8). Tumor angiogenesis is a well-known pathway that mediates the 
distant metastasis of cancer cells (9). Zeng et al demonstrated that LACTB attenuated p53 ubiquitination and inhibited the progression of colorectal cancer (10). However, in contrast, LACTB was found to contribute to cancer metastasis and to correlate with the poor prognosis of nasopharyngeal and pancreatic cancer $(11,12)$. These data suggest that the role of LACTB differs in a particular cancer. Chemotherapy greatly improved the prognosis of cancer patients in the clinic. However, drug resistance is now a great challenge in the clinic. Drug resistance often reduces the efficacy of chemotherapeutic drugs and results in short survival and low quality of life of patients (13). LACTB was shown to induce apoptosis of oxaliplatin-resistant cells and improve the efficacy of oxaliplatin in gastric cancer (14). What is the role of LACTB in lung cancer therapy and its underlying mechanism?

Epithelial-mesenchymal transition (EMT) is an important cellular program and is responsible for the aggressive behavior in cancer (15). Activation of the EMT process confers increased metastatic potential and drug resistance to cancer cells. In lung cancer, activation of the EMT signaling pathway greatly promotes tumor progression (16). At the molecular level, LACTB was reported to inhibit EMT and suppress tumor progression in colorectal cancer (17). Based on the information, it is thought that LACTB might regulate progression of lung cancer through EMT signaling.

In the present study, to explore the role of $L A C T B$ in lung cancer, the expression of $L A C T B$ was overexpressed/knocked down and lung cancer cells were subsequently treated with docetaxel. Then, cell invasive ability, apoptosis and the mechanism of action were determined.

\section{Materials and methods}

Bioinformatics analysis. The expression and methylation levels of LACTB in lung cancer were obtained by analyzing data from The Cancer Genome Atlas (TCGA) database (http://ualcan.path.uab.edu/analysis.html). The association of LACTB expression with the survival of lung cancer patients was obtained from the public database Kaplan-Meier Plotter (http://kmplot.com/analysis/index. $\mathrm{php}$ ? $\mathrm{p}=$ service \&cancer=lung).

Cell culture. H1299 (non-small cell lung cancer) and H1975 (lung adenocarcinoma) cell lines were purchased from the Chinese Academy of Sciences (Shanghai, China) and cultured in RPMI-1640 medium (Gibco; Thermo Fisher Scientific, Inc.) containing $10 \%$ fetal bovine serum (FBS; Gibco; Thermo Fisher Scientific, Inc.) at $37^{\circ} \mathrm{C}$. The culture medium was refreshed every day and the cells were passaged at 1:3 after arriving at $100 \%$ confluence.

Reverse-transcription-quantitative polymerase chain reaction ( $q P C R)$. Total RNA was extracted from the treated cell lines with RNeasy kit (Beyotime Institute of Biotechnology) according to the manufacturer's instructions. One microgram of total RNA was used as template for the synthesis of the first strand of cDNA with a cDNA synthesize kit (Beyotime Institute of Biotechnology). Then $1 \mu \mathrm{l}$ of cDNA was used as a template to quantify the expression of target genes with SYBRGreen Mix (Beyotime Institute of Biotechnology). The protocol was as follows: $95^{\circ} \mathrm{C}$ for $5 \mathrm{~min} ;\left(95^{\circ} \mathrm{C}\right.$ for $15 \mathrm{sec} ; 60^{\circ} \mathrm{C}$ for $15 \mathrm{sec}$ ) for 40 cycles. Then the $2^{-\Delta \Delta \mathrm{Cq}}$ method was used to analyze the relative expression of target genes (18). GAPDH was used as the internal control. The primers for qPCR are shown in Table I.

Western blot analysis. Total protein was extracted from the treated cell lines with Membrane and Cytosol Protein Extraction Kit (Beyotime Institute of Biotechnology) according to the manufacturer's instructions. Total protein $(10 \mu \mathrm{g})$ was separated by SDS-PAGE method and transferred onto PVDF membranes (Beyotime Institute of Biotechnology). Then the PVDF membranes were blocked with 5\% non-fat milk (Beyotime Institute of Biotechnology) for $1 \mathrm{~h}$ at room temperature followed by incubation with primary antibodies against the target protein at $4^{\circ} \mathrm{C}$ overnight. After washing with TBST (Beyotime Institute of Biotechnology) for three times, the PVDF membranes were incubated with secondary antibodies for $1 \mathrm{~h}$ at room temperature. After washing with TBST for three times, the ECL kit (Beyotime Institute of Biotechnology) was used to detect the protein bands on the PVDF membranes. $\beta$-actin was used as the internal control. The primary and secondary antibodies were as below: MMP9 (cat. no. 10375-2-AP; dilution, 1:500), MMP2 (cat. no. 10373-2-AP; dilution, 1:500), N-cadherin (cat. no. 22018-1-AP; dilution, 1:5,000), E-cadherin (cat. no. 20874-1-AP; dilution, 1:5,000), SNAIL (cat. no. 13099-1-AP; dilution: 1:500), LACTB (cat. no. 18195-1-AP; dilution, 1:2,000), Vimentin (cat. no. 10366-1-AP; dilution, 1:5,000), GAPDH (cat. no. 10494-1-AP; dilution, 1:10,000) and Rabbit IgG (cat. no. SA00001-2; dilution, 1:5,000)(all ProteinTech Group, Inc.). Adobe Photoshop CS6 (Adobe Systems, Inc.) was used for quantification of the blots.

Synthesis of shRNA targeting LACTB, construction of the plasmid carrying the LACTB gene and drug administration. shRNA targeting the human $L A C T B$ gene (shLACTB) was designed and synthesized, and the negative control was shNC. The target sequences are listed in Table II. Then 40-80 pmol of shLACTB or shNC was used to transfect them into the tumor cells with Lipo2000 agent (Invitrogen; Thermo Fisher Scientific, Inc.) for $6 \mathrm{~h}$. Then fresh medium was added into each well and cultured for another $48 \mathrm{~h}$ followed by subsequent experiments.

Alentiviral expression plasmid carrying the coding sequence of the human LACTB gene (pLACTB) was constructed, confirmed by sequencing and the lentivirus-expressing LACTB was prepared by Shanghai GeneChem. Then, $2 \mu 1$ of the plasmid was added into the cancer cells. After culture for $72 \mathrm{~h}$, subsequent experiments were carried out.

Cells were treated with the designed dosage of docetaxel (10, 50,100 and $200 \mathrm{nmol} / \mathrm{l})$ (QILU Pharmaceutical Co.,Ltd.) in combination with shNC, shLACTB or empty vector pCDH (Ctrl) or pLACTB for $48 \mathrm{~h}$ followed by subsequent experiments.

Cell Counting Kit 8 (CCK-8). A total of $3 \times 10^{3}$ cells/well were seeded into 96 -well plates and treated as described above. After culture for $72 \mathrm{~h}, 10 \%$ of CCK- 8 agent (Yeasen) was added into 
Table I. Primers for quantitative polymerase chain reaction assay.

\begin{tabular}{lc}
\hline Gene & \multicolumn{1}{c}{ Primers $\left(5^{\prime}-3^{\prime}\right)$} \\
\hline$L A C T B$ & \\
Forward & GTGGTTGGAGTTTCTGTAGATGGAA \\
Reverse & AGTAATCTTGTTGTGACAGAAACCT \\
GAPDH & \\
Forward & CGTATTGGGCGCCTGGTCAC \\
Reverse & ATGATGACCCTTTTGGCTCC \\
\hline
\end{tabular}

$L A C T B$, lactamase $\beta$.

Table II. Target sequence for $\angle A C T B$ gene.

\begin{tabular}{ll}
\hline Gene & \multicolumn{1}{c}{ Targets (5'-3') } \\
\hline LACTB & \\
shRNA-\#1 & CCTTACGTGGATAACTCCTAT \\
shRNA-\#2 & CCTTAACACCATAGGTGCAAA \\
shNC & CCTTCGCCTAAGCATTAGGCAT \\
\hline
\end{tabular}

$L A C T B$, lactamase $\beta$.

each well and cultured for another $1 \mathrm{~h}$. Then, the absorbance at $450 \mathrm{~nm}$ was detected on a microplate reader (Tecan-SPARK).

Wound-healing assay. A total of $2 \times 10^{5}$ cells/well were seeded into 24-well plates and treated as described above. After culture for $24 \mathrm{~h}$, the confluence of $90 \%$ was achieved, and a scratch was produced using a $10-\mu 1$ tip followed by washing with PBS (Gibco; Thermo Fisher Scientific, Inc.) two times. The width of the scratch was determined and recorded as $\mathrm{S}_{0 \mathrm{~h}}$. After culture for another $24 \mathrm{~h}$, the width of each scratch was determined and recorded as $S_{48} \mathrm{~h}$. The relative migration rate was calculated as follows: Migration rate $=\left(\mathrm{S}_{0 \mathrm{~h}}-\mathrm{S}_{24 \mathrm{~h}}\right) / \mathrm{S}_{0 \mathrm{~h}}$.

Transwell assay. The insert chamber with $8-\mu \mathrm{m}$ pores (Corning, Inc.) was pretreated with/without $200 \mu \mathrm{l}$ of cold Matrigel (BD, Bioscience) at room temperature for $1 \mathrm{~h}$ followed by washing with culture medium once. Then a total of $2 \times 10^{4}$ cells/well were added to each room with $300 \mu 1$ of culture medium containing no serum and the room was inserted into 24-well plates with $300 \mu \mathrm{l}$ of culture medium containing $10 \% \mathrm{FBS}$. After culture for $24 \mathrm{~h}$, cells on the upper surface of the insert room were scraped and cells on lower surface was fixed in $4 \%$ paraformaldehyde (Beyotime Institute of Biotechnology) for $10 \mathrm{~min}$ at room temperature. After washing with PBS, the insert chamber was stained with $0.5 \%$ crystal violet (Beyotime Institute of Biotechnology) for $10 \mathrm{~min}$ at room temperature. Then the positive cells were counted under a light microscope (Olympus) at x100 magnification after washing with PBS.

Flow cytometric analysis. A total of $2 \times 10^{5}$ cells/well were seeded into 6-well plates and treated as the above. After culture for $48 \mathrm{~h}$, cells were collected and washed with cold PBS once. Then cells were suspended in staining buffer and stained with Annexin V-PE/7-AAD reagent (Beyotime Institute of Biotechnology) at room temperature for $15 \mathrm{~min}$ in the dark. The positively marked cells were detected with BD FACSCelesta (BD Biosciences).

Statistical analysis. The data in this study were analyzed using SPSS software (SPSS, Inc.; Version 16.0) and was expressed as the mean $\pm \mathrm{SD}$. The difference between two groups was analyzed by unpaired Student's t-test, while one-way ANOVA followed by Tukey's test was used for more than three groups. $\mathrm{P}<0.05$ indicates a significant difference.

\section{Results}

LACTB is correlated with favorable prognosis in lung cancer. To study the role of the LACTB gene in lung cancer, we extracted information from public databases. Based on the TCGA database, the expression level of LACTB in 515 tissues with lung cancer was significantly downregulated compared to that in the normal tissues (Fig. 1A). The methylation level of LACTB in tumor tissues was significantly higher than in normal control tissues (Fig. 1B). Moreover, survival analysis demonstrated that the survival probability of patients with high LACTB expression was significantly better than that of patients with low LACTB expression (Fig. 1C). These data suggest that LACTB might play an important role in the progression of lung cancer.

LACTB does not affect cell survival in lung cancer. To investigate the role of LACTB in lung cancer, the expression of LACTB was increased or decreased in the H1299 and H1975 cell lines. As shown in Fig. 2A and B, qPCR analysis showed that LACTB was successfully increased in the H1299 and H1975 cells. Additionally, shRNA technology ensured that LACTB was significantly decreased (Fig. 2C and D) in the H1299 and H1975 cells. Then by CCK-8 assay, cell survival was shown to be minimally affected by LACTB (Fig. 2E and F). Therefore, LACTB does not affect cell survival in lung cancer.

LACTB negatively regulates migration and invasion in lung cancer. By wound-healing assay, we found that the cell migration rate was greatly reduced by LACTB overexpression in the H1299 and H1975 cells. As shown in Fig. 3A and B, the relative cell migration rates were 0.31 and 0.12 after LACTB overexpression in H1299 and H1975 cells, respectively, which were significantly lower than those of the control group. However, in H1299 cells with LACTB knockdown, the migration rate was 0.81 while it was 0.44 in the NC group (Fig. 4A and B). The same trend was observed in the H1975 cells. In Figs. 5A and B, and 6A and B, Transwell assays without Matrigel demonstrated that the cell migration was significantly suppressed by LACTB overexpression but promoted by LACTB knockdown. Then, in the Transwell assay with Matrigel, there were fewer cells penetrating through the monolayer membrane in the LACTB overexpression group than in the control group in H1299 cells (18 vs. 99; Fig. 7A 
A

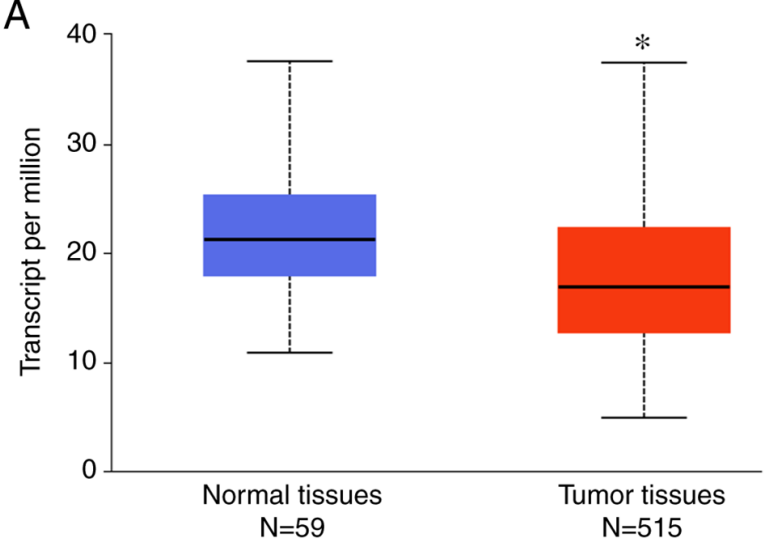

B

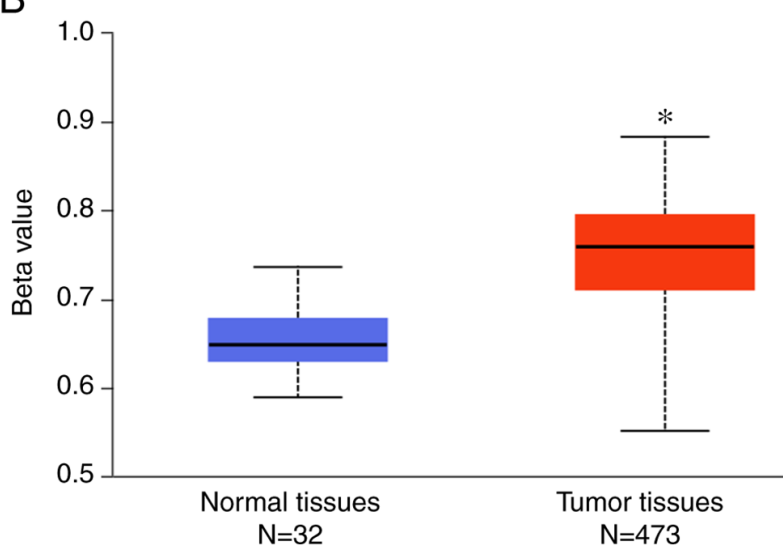

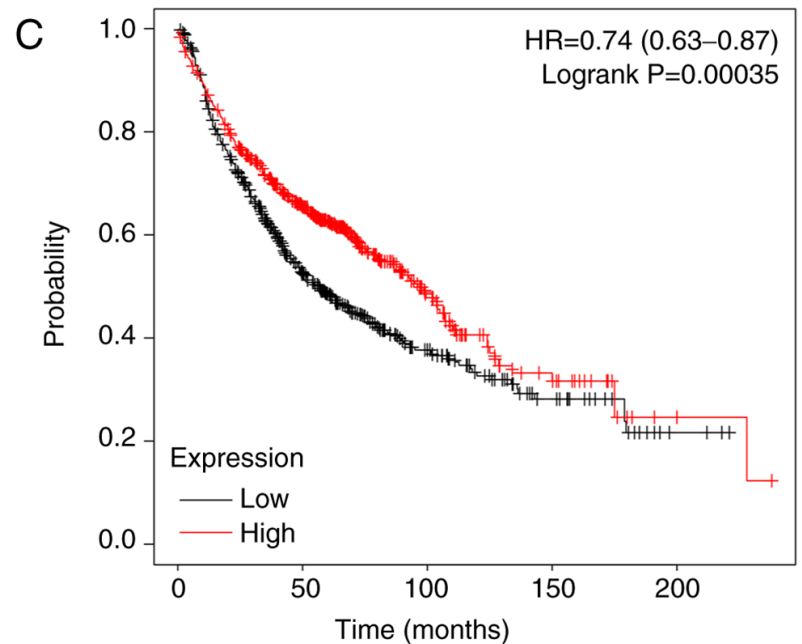

Figure 1. LACTB is clinically associated with lung cancer. (A) LACTB was found to be significantly decreased in lung cancer tissues compared to that noted in normal tissues based on TCGA database. (B) The methylation level of LACTB in lung cancer tissues was increased significantly compared to normal tissues based on the TCGA database. (C) The survival probability of lung cancer patients with high LACTB expression was much higher than the patients with low LACTB expression. "P<0.05, statistical difference compared to the normal tissues. LACTB, lactamase $\beta$; TCGA, The Cancer Genome Atlas.

A

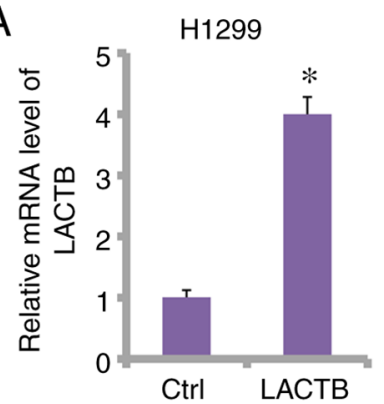

D

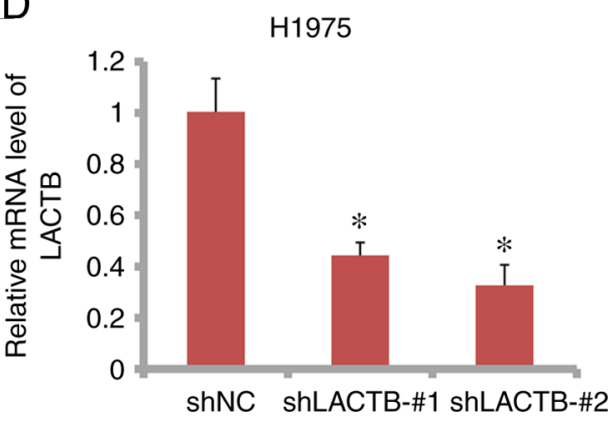

B

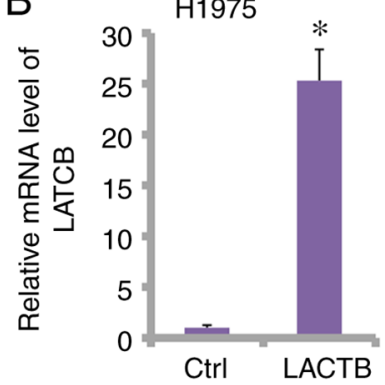

E

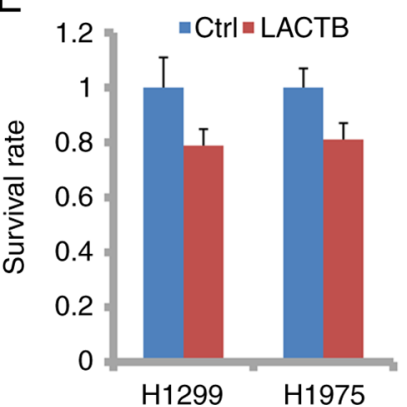

C

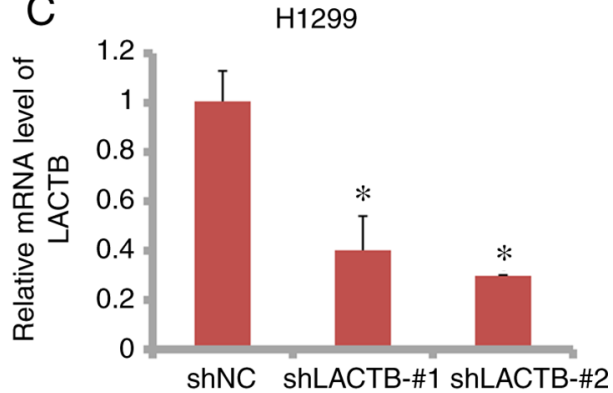

F

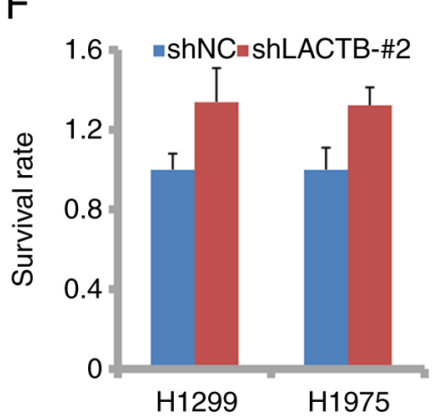

Figure 2. LACTB negatively regulates the cell survival in lung cancer. (A) LACTB was found to be overexpressed in H1299 cells by transfection with a plasmid carrying LACTB. In the control group (Ctrl), empty vector pCDH was transfected into cells. (B) LACTB was overexpressed in H1975 cells by transfection with a plasmid carrying LACTB. (C) LACTB was knocked down in H1299 cells by shRNA targeting LACTB. (D) LACTB was knocked down in H1975 cells by shRNA targeting LACTB. (E) Overexpression of LACTB inhibited cell survival in H1299 and H1975 cells. (F) Knockdown of LACTB promoted cell survival in $\mathrm{H} 1299$ and H1975 cells. ${ }^{*} \mathrm{P}<0.05$, statistical difference compared with the Ctrl or shNC group. LACTB, lactamase $\beta$. 
A

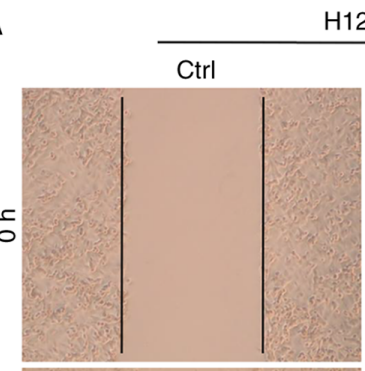

$\mathrm{H} 1299$

$\mathrm{H} 1975$
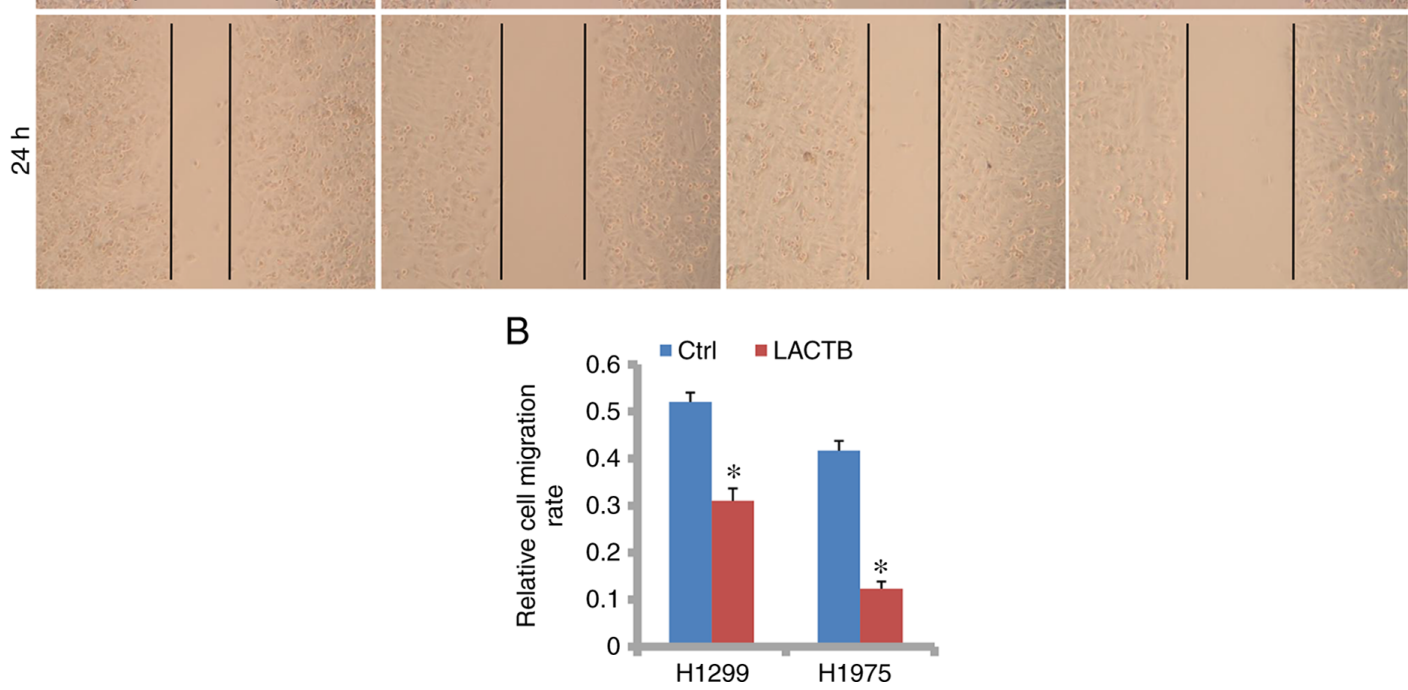

Figure 3. Overexpression of LACTB suppresses lung cancer cell migration in a wound-healing assay. (A) Cell migration behavior was determined by a wound-healing assay in $\mathrm{H} 1299$ and H1975 cells. (B) The statistical analysis of data in (A). ${ }^{*} \mathrm{P}<0.05$, statistical difference compared with the Ctrl group. LACTB, lactamase $\beta$.

A

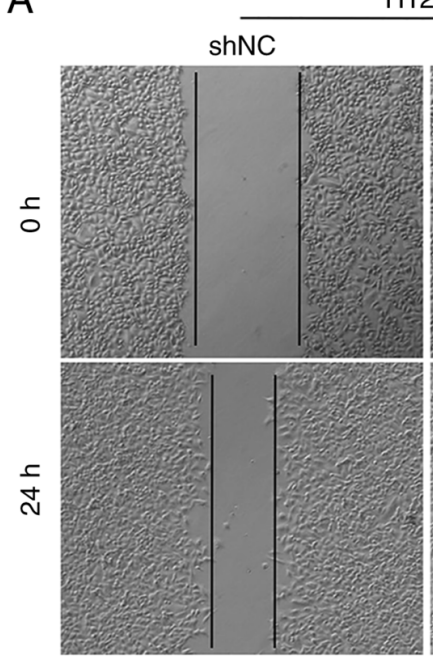

$\mathrm{H} 1299$

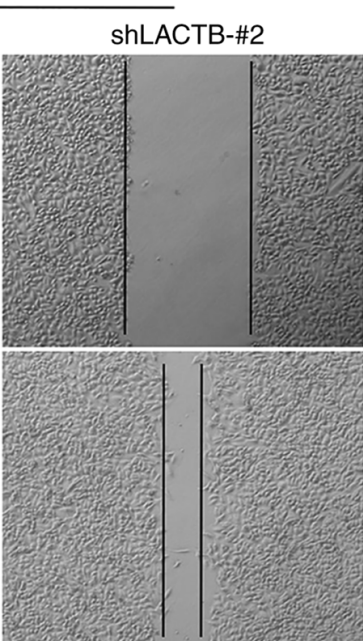

$\mathrm{H} 1975$

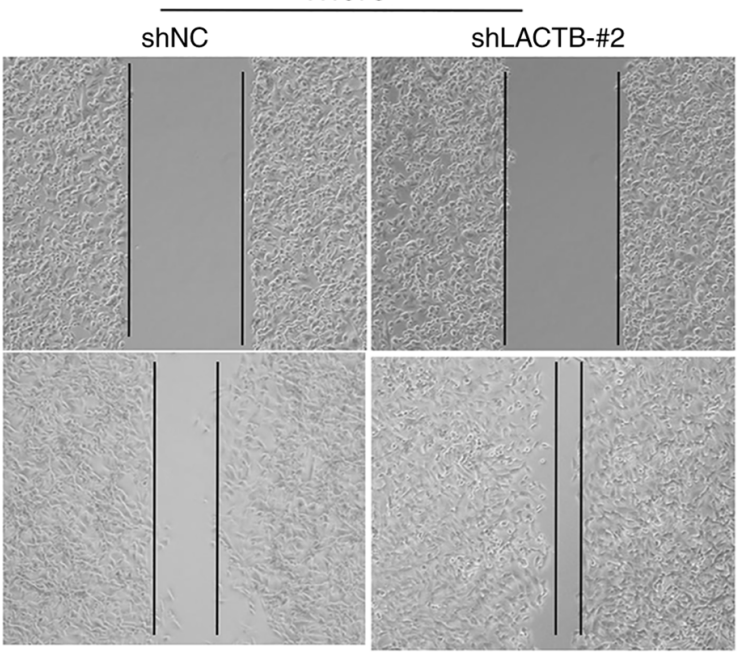

B

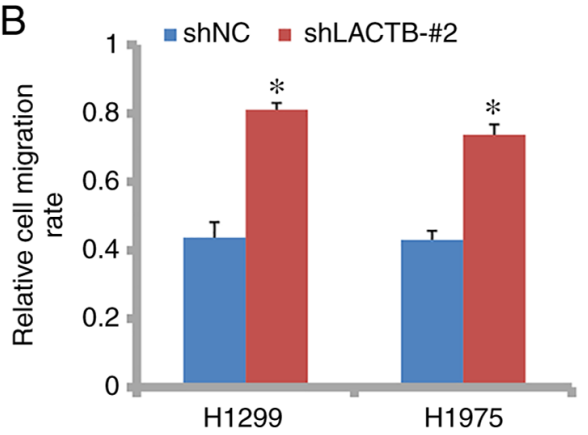

Figure 4. Knockdown of LACTB promotes lung cancer cell migration in a wound-healing assay. (A) Cell migration behavior was determined by a wound-healing assay in $\mathrm{H} 1299$ and $\mathrm{H} 1975$ cells. (B) The statistical analysis of data in (A). ${ }^{*} \mathrm{P}<0.05$, statistical difference compared to the shNC group. LACTB, lactamase $\beta$. 
A

A

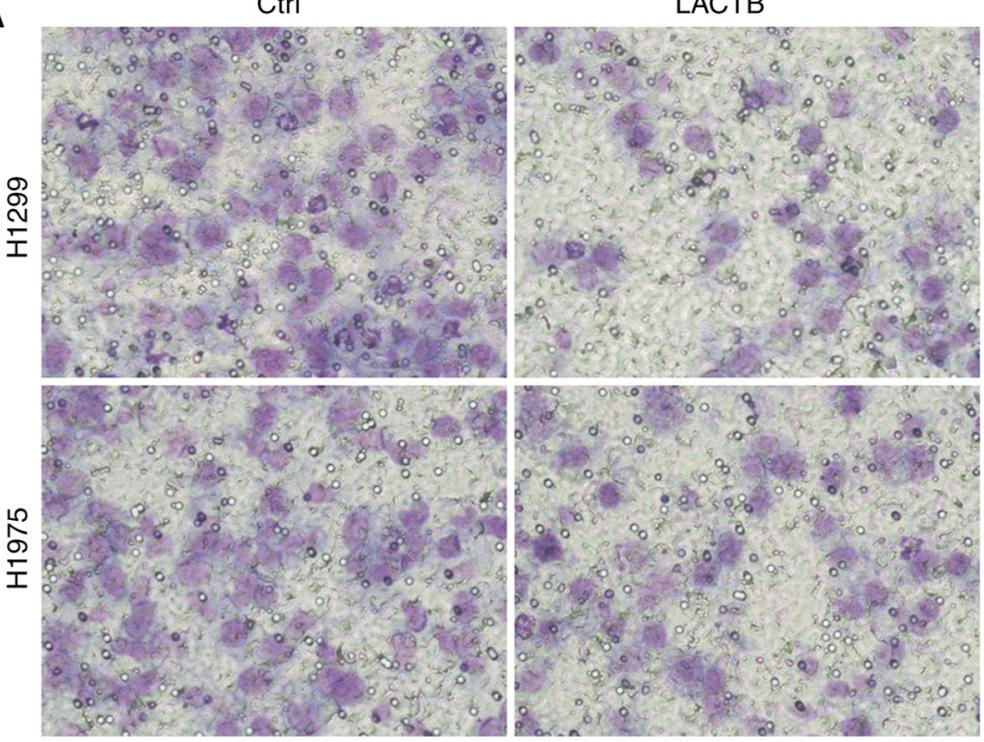

B

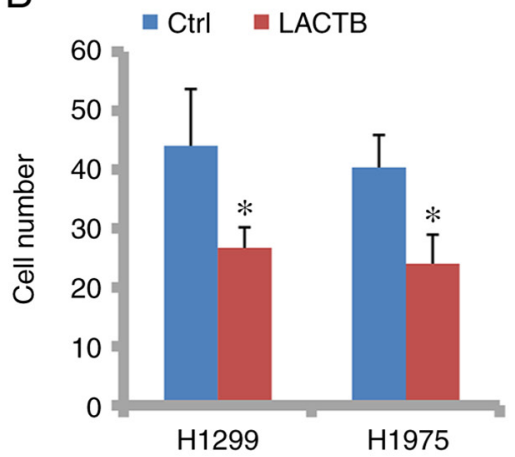

Figure 5. Overexpression of LACTB suppresses lung cancer cell migration in a Transwell assay. (A) Cell migration behavior was determined by a Transwell assay in H1299 and H1975 cells. (B) The statistical analysis of data in (A). * P<0.05, statistical difference compared to the Ctrl group. LACTB, lactamase $\beta$.

A

ஓ्

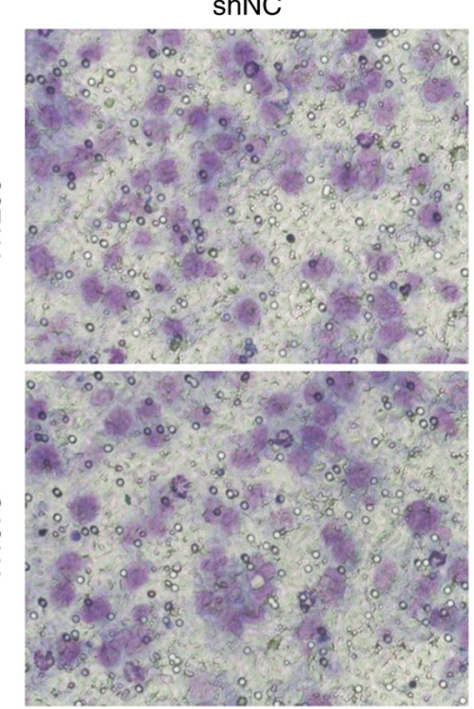

shLACTB-\#2

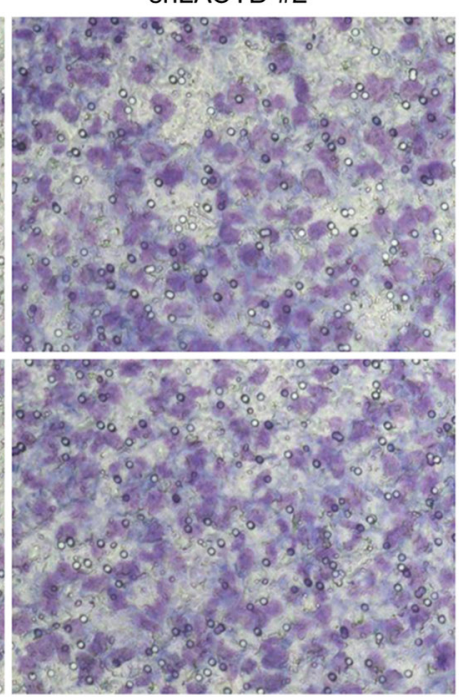

B m shNC $=$ shLACTB-\#2

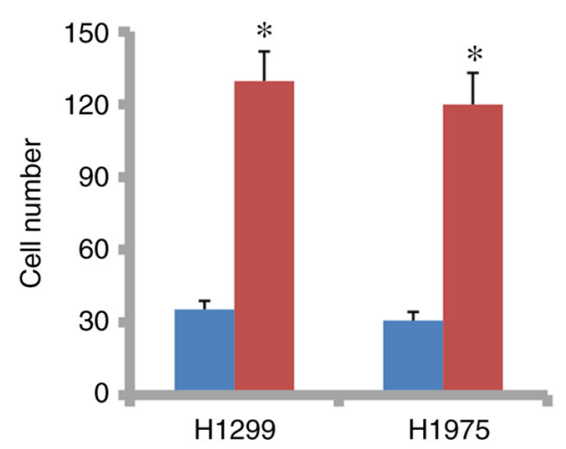

Figure 6. Knockdown of LACTB promotes lung cancer cell migration in a Transwell assay. (A) Cell migration behavior was determined by a Transwell assay in $\mathrm{H} 1299$ and $\mathrm{H} 1975$ cells. (B) The statistical analysis of data in (A). ${ }^{*} \mathrm{P}<0.05$, statistical difference compared to the shNC group. LACTB, lactamase $\beta$.

and B). However, in contrast, LACTB knockdown significantly increased the cell number (Fig. 8A and B). Therefore, LACTB negatively regulates cell migration and invasion abilities in lung cancer cells.

LACTB increases the inhibitory effect of docetaxel on cell proliferation. The combination of drugs in cancer is often surprising. Here, we found that LACTB overexpression significantly inhibited the cell proliferation in H1299 and H1975 cells treated with docetaxel, which indicates that LACTB has additive effects with docetaxel (Fig. 9A and B). In contrast, knockdown of LACTB greatly reduced the inhibitory ability of docetaxel on lung cancer cells (Fig. 9C and D). These data suggest that LACTB might be applied to increase the efficacy of docetaxel in lung cancer therapy.
LACTB induces apoptosis of lung cancer cells induced by docetaxel. Docetaxel induces cell apoptosis in cancer. As shown in Fig. 10A and B, the cell apoptotic rate was $48.96 \%$ in the H1975 cells treated with the combination of LACTB and docetaxel while the rate was $33 \%$ in the Ctrl/docetaxel group, and the difference was significant. However, LACTB knockdown reduced the apoptotic rate of docetaxel in the H1975 cells (Fig. 11A and B). The apoptotic rate in the shNC/docetaxel group was $53.86 \%$ while it was $26.84 \%$ in the shLACTB/docetaxel group. In conclusion, LACTB plays an additive role with docetaxel in the apoptosis of lung cancer cells.

$L A C T B$ regulates the expression of members of the EMT signaling pathway. By western blot analysis, we detected obvious changes in typical molecules in the EMT process. As 

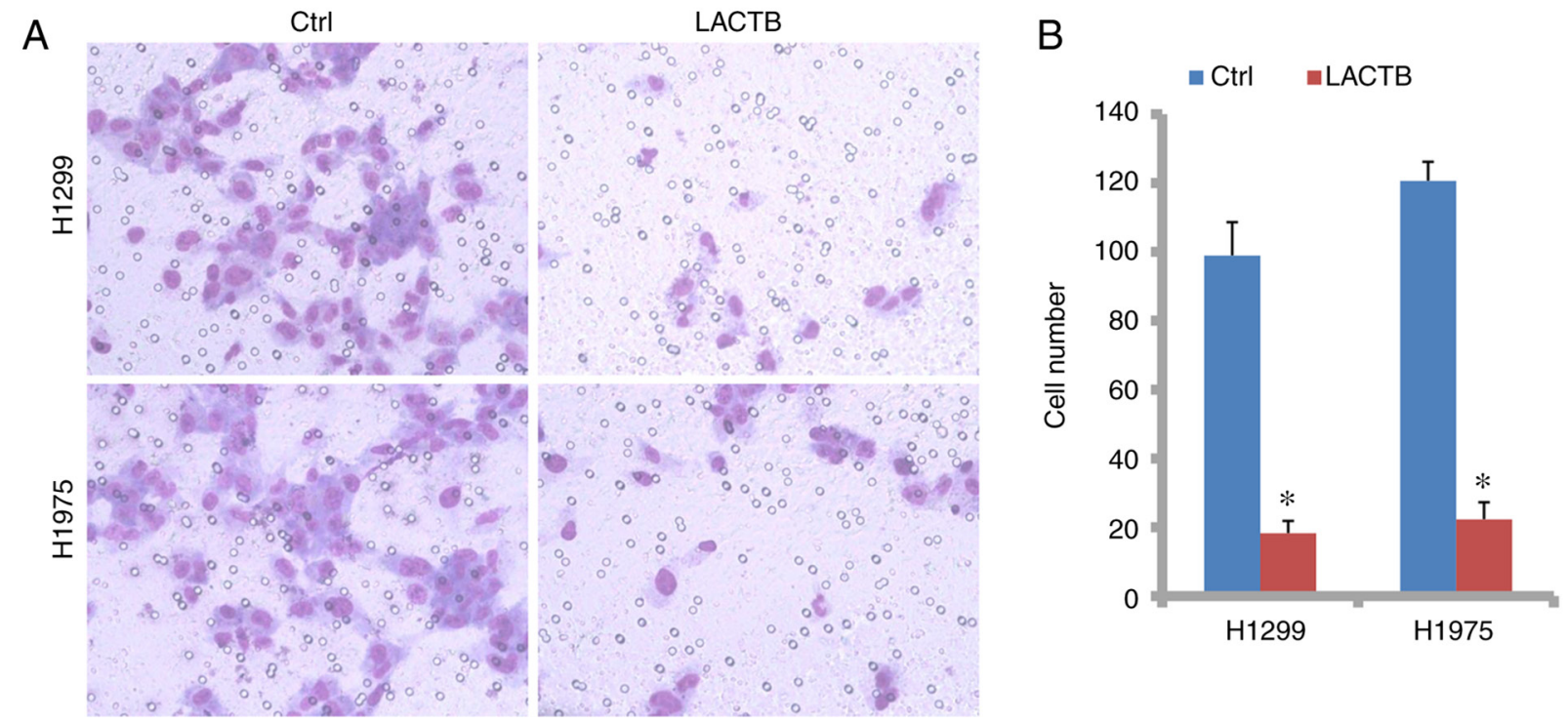

Figure 7. Overexpression of LACTB suppresses cell invasion in lung cancer. (A) Cell invasion behavior was determined by a Transwell assay in H1299 and H1975 cells. (B) The statistical analysis of data in (A). ${ }^{*} \mathrm{P}<0.05$, statistical difference compared with the Ctrl group. LACTB, lactamase $\beta$.
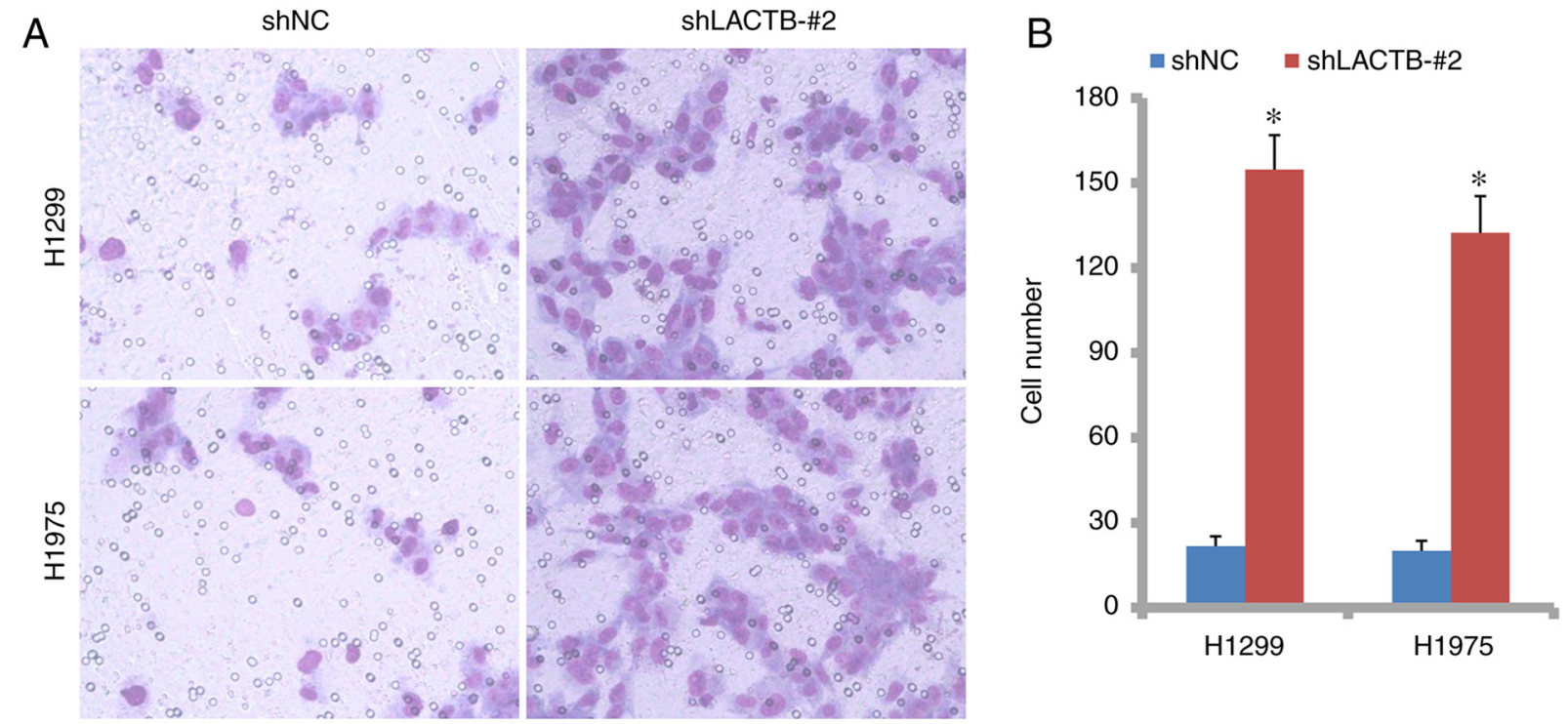

Figure 8. Knockdown of LACTB promotes cell invasion in lung cancer. (A) Cell migration behavior was determined by a Transwell assay in H1299 and H1975 cells. (B) The statistical analysis of data in (A). " $\mathrm{P}<0.05$, statistical difference compared to the shNC group. LACTB, lactamase $\beta$.

shown in Fig. 12A and B, the expression levels of Snail, matrix metalloproteinase (MMP2), MMP9, N-cadherin and vimentin were significantly downregulated while E-cadherin was significantly increased after LACTB overexpression in H1975 cells. Conversely, knockdown of LACTB reduced E-cadherin levels while increasing the levels of Snail, MMP2, MMP9, $\mathrm{N}$-cadherin and vimentin (Fig. 12A and C). This indicates that LACTB negatively regulates the EMT process in lung cancer.

\section{Discussion}

Thousands of humans succumb to lung cancer each year, and overall survival is a major challenge in the clinic $(1,2)$. This is due to the lack of knowledge concerning lung cancer including how and when lung cancer begins or progresses.
Based on TCGA database (19), lactamase $\beta$ (LACTB) was shown to be downregulated in lung cancer while the methylation level of LACTB was enhanced. Methylation is an important mechanism regulating the switch-on/off of target genes (20). Reduced expression of LACTB in lung cancer tissues might be due to increased methylation levels of LACTB. In colorectal cancer, LACTB was also found to be silenced by elevated methylation of the promoter domain, which promoted metastasis and advanced clinical stages (10). In the Kaplan-Meier Plotter database, high LACTB expression was associated with better survival of lung cancer patients. This is consistent with a study in hepatocellular cancer that found that low LACTB expression was associated with poor prognosis (21). These data suggest that LACTB might also be a suppressor in lung 

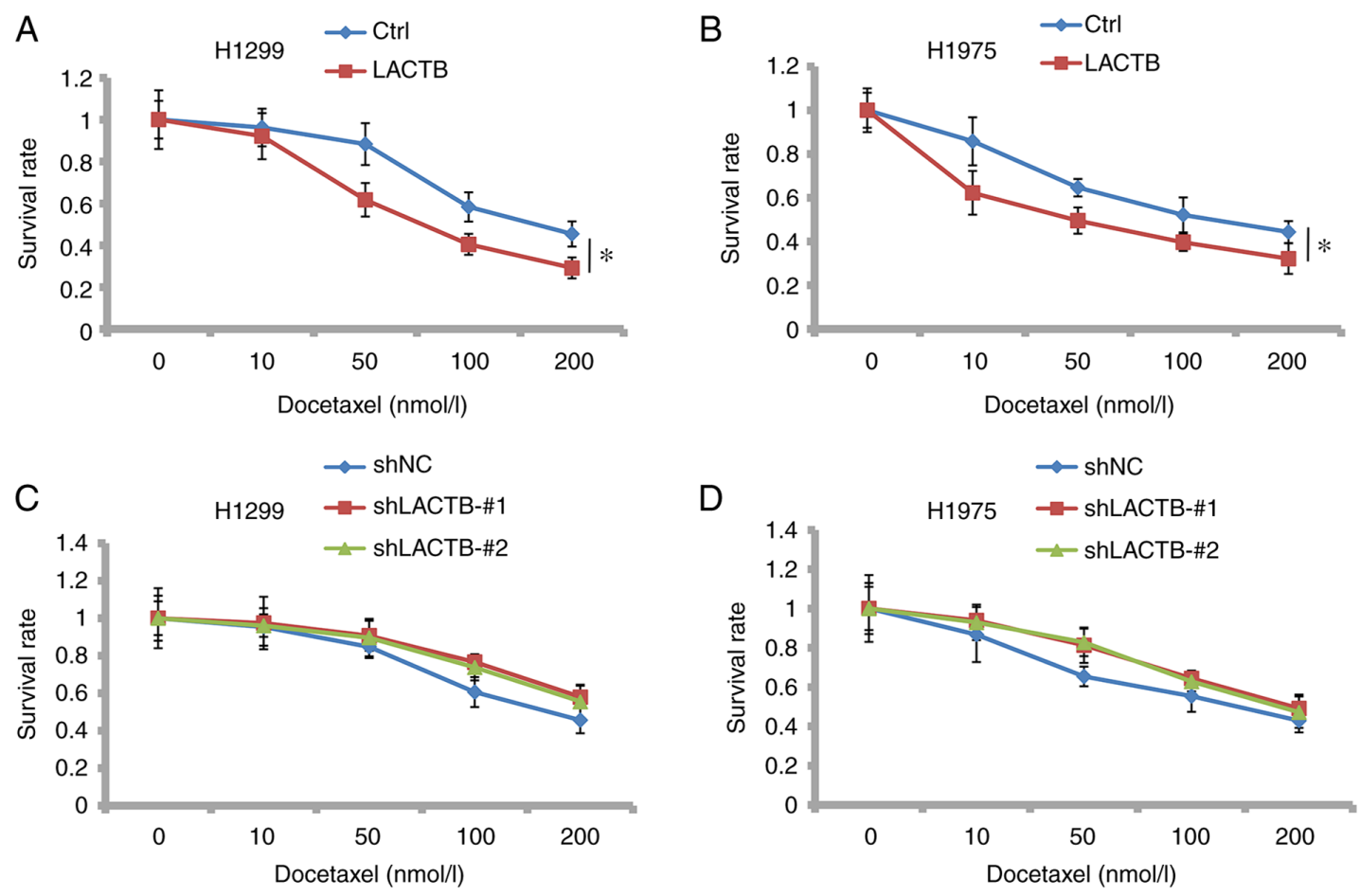

Figure 9. LACTB promotes the cell killing effect of docetaxel on lung cells. (A) Combination of LACTB with docetaxel further reduced the survival rate of $\mathrm{H} 1299$ cells. (B) Combination of LACTB with docetaxel further reduced the survival rate of H1975 cells. (C) Knockdown of LACTB partially reversed the growth inhibition of $\mathrm{H} 1299$ cells by docetaxel. (D) Knockdown of LACTB partially reversed the growth inhibition of H1975 cells by docetaxel. "P<0.05, statistical difference. LACTB, lactamase $\beta$
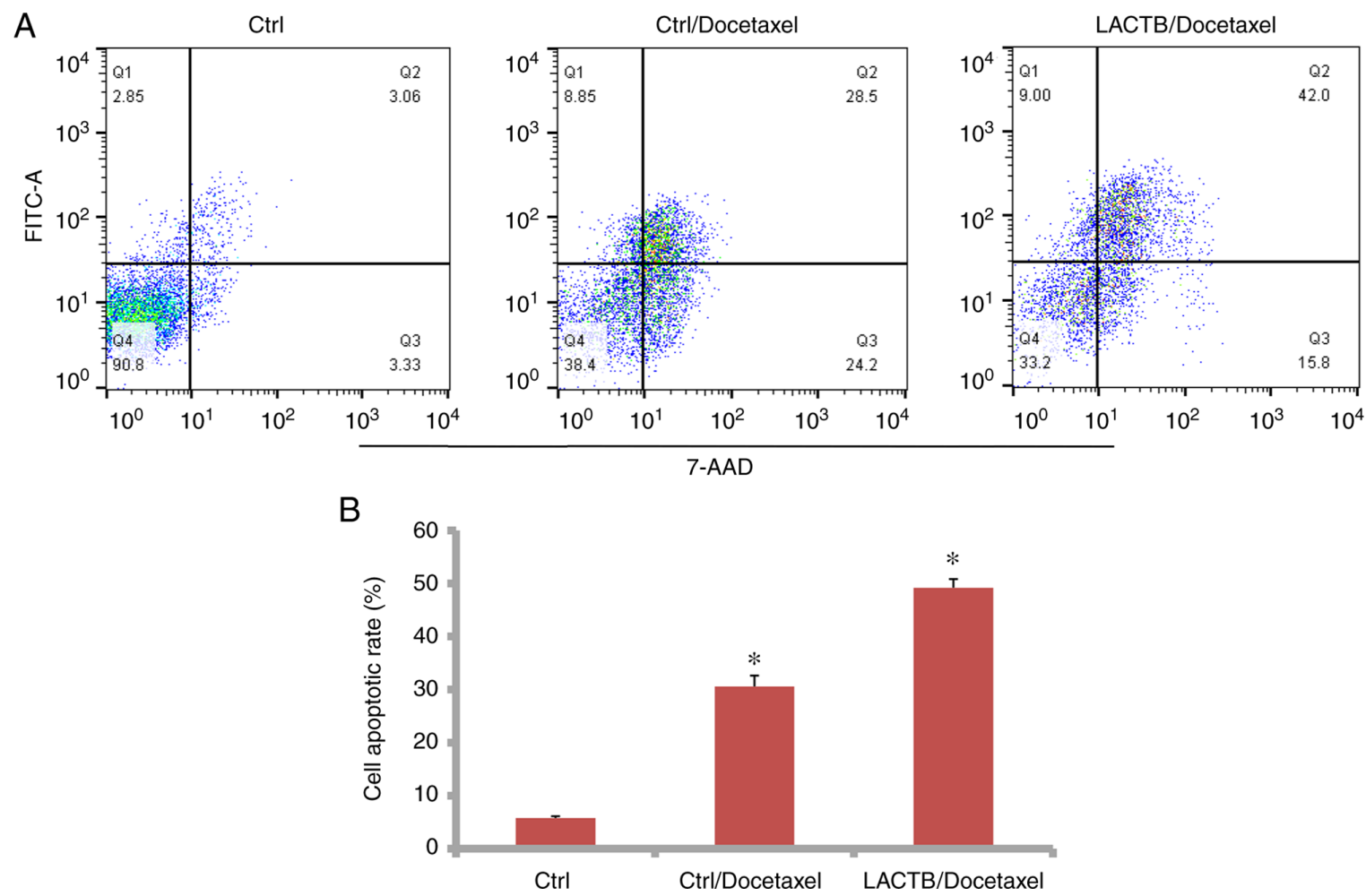

Figure 10. Overexpression of LACTB enhances the cell apoptotic rate by docetaxel in H1975 cells. (A) Cell apoptosis rate in H1975 cells was detected by FACS analysis. (B) The statistical analysis of data in (A). ${ }^{*} \mathrm{P}<0.05$, statistical difference compared with the Ctrl group. LACTB, lactamase $\beta$.

cancer and a potential risk factor when evaluating the prognosis of lung cancer patients. In the present study, overexpression of LACTB was found to suppressed cell proliferation, migration and invasion in lung cancer cells. However, knockdown of LACTB reversed the inhibitory effect in lung cancer. Generally, expansion of tumors and 
A

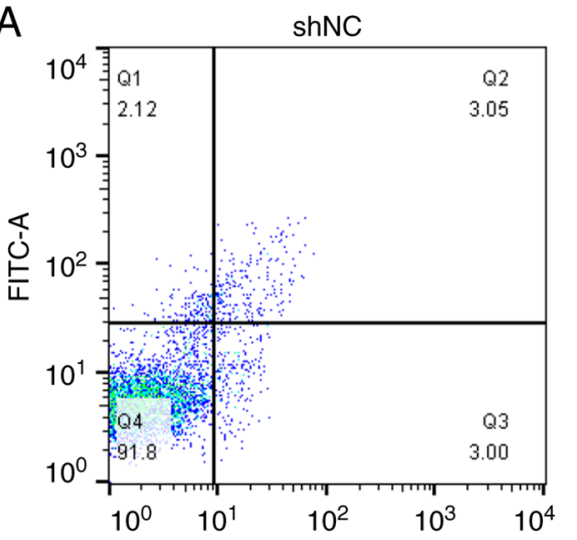

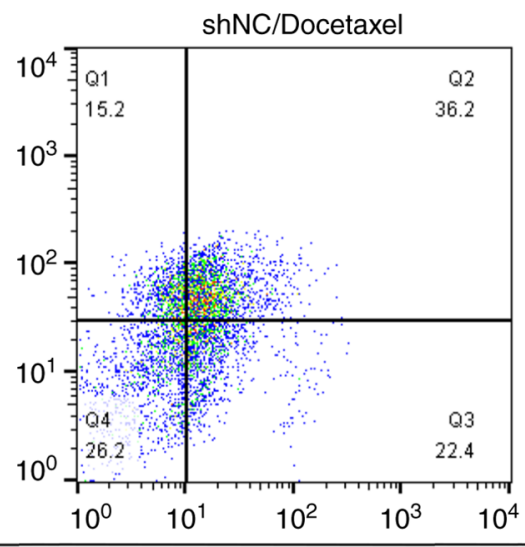

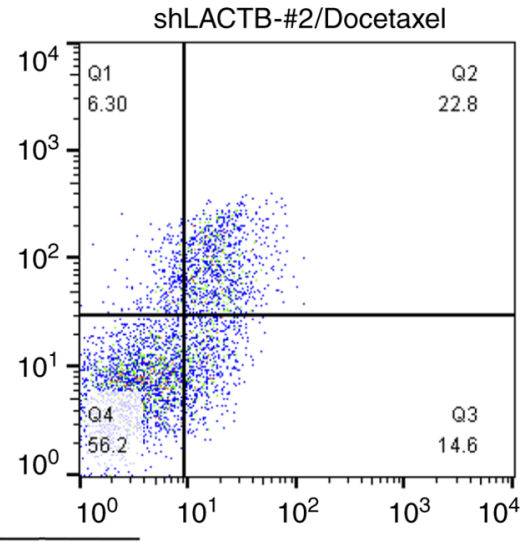

B

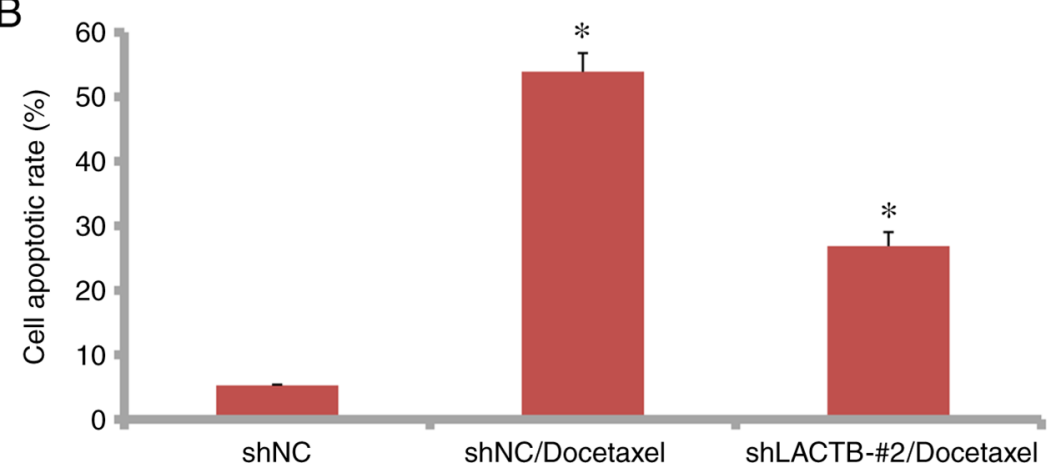

Figure 11. Knockdown of LACTB reduces the cell apoptotic rate by docetaxel in H1975 cells. (A) Cell apoptosis rate in H1975 cells was detected by FACS analysis. (B) The statistical analysis of data in (A). ${ }^{*} \mathrm{P}<0.05$, statistical difference compared to the shNC group. LACTB, lactamase $\beta$.

A

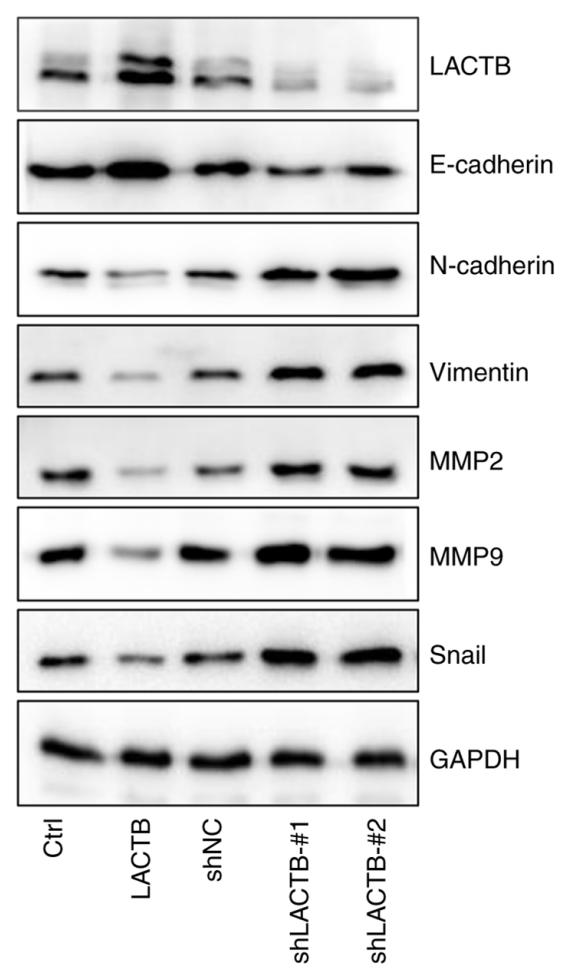

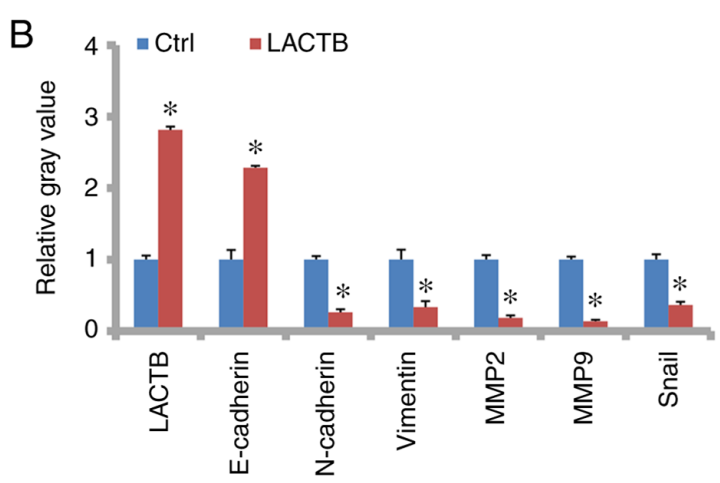

C

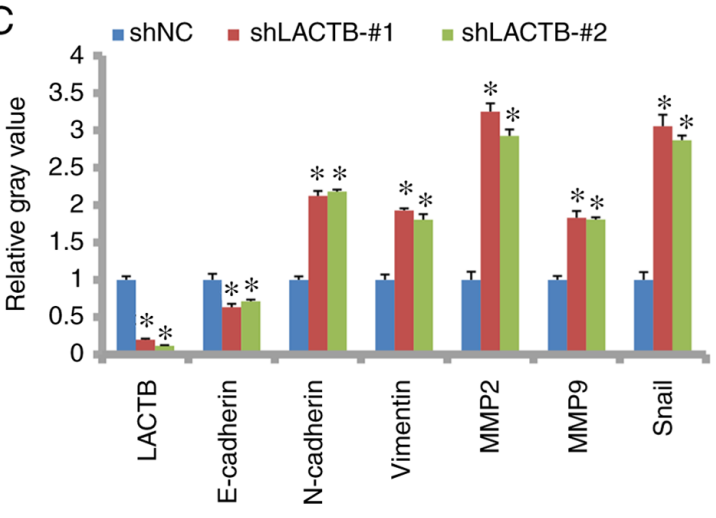

Figure 12. LACTB regulates the EMT signaling pathway. (A) Overexpression of LACTB promoted E-cadherin expression and reduced the level of Snail, N-cadherin, vimentin, MMP2 and MMP9 in H1975 cells. By contrast, knockdown of LACTB increased the level of Snail, N-cadherin, vimentin, MMP2 and MMP9 while reducing E-cadherin in H1975 cells. (B) Gray value analysis of the protein bands (on the left) shown in in A. (C) Gray value analysis of the protein bands (on the right) in (A). ${ }^{*} \mathrm{P}<0.05$, statistical difference compared to the $\mathrm{Ctrl}$ or shNC group. LACTB, lactamase $\beta$; EMT, epithelial-mesenchymal transition; MMP, matrix metalloproteinase. 
distant metastasis are two typical events observed in cancer patients (22). This study confirmed the suppressor role of LACTB in lung cancer.

Docetaxel is a specific inhibitor of the M-phase of the cell cycle and has been successfully applied in the clinic to treat patients with breast cancer or non-small cell lung cancer. Docetaxel was demonstrated to increase the ratio of Bax to Bcl-2 followed by the apoptosis response when combined with Stattic in prostate cancer (23). In another study, docetaxel enhanced lysosomal function followed by activated autophagy (24). In the present study, docetaxel induced apoptosis in lung cancer and this effect was reinforced by LACTB overexpression. This suggests that synergic or additive effects between docetaxel and LACTB exist. LACTB might increase the efficacy of docetaxel or may allow a decrease in the dosage of docetaxel, which would relieve the adverse effects of docetaxel. Thus, combination therapy could achieve much better outcomes and fewer adverse effects. A potential combination therapy could be the combination of immunotherapy with radiation therapy or targeted therapy $(25,26)$. Therefore, LACTB has the clinical potential to increase the efficacy of docetaxel.

EMT is critical in cancer transformation and confers aggressive ability to cancer cells (27). In lung cancer, activation of EMT was reported to cause a series of events favorable for cancer progression. For example, the efficacy of EGFR-targeted therapy in lung cancer was greatly reduced through the EMT mechanism (28). T-cell infiltration in lung cancer was negatively regulated by EMT (29). In the present study, LACTB exhibited potent inhibition of cell migration and invasion in lung cancer. At the molecular level, LACTB was shown to regulate the expression levels of E-cadherin, $\mathrm{N}$-cadherin, vimentin and Snail. E-cadherin is a typical marker of epithelial cells while $\mathrm{N}$-cadherin and vimentin are typical markers of mesenchymal cells (30). Snail was reported to bind to the E-box element in the promoter of the E-cadherin gene and suppress the transcription of E-cadherin (31). Therefore, LACTB suppressed EMT in lung cancer. The inhibitory role of LACTB on cell migration or invasion might be partially due to the deficiency of EMT in lung cancer. EMT was also shown to cause drug resistance to docetaxel in lung cancer therapy (32). In this study, LACTB suppressed the EMT process and induced cell apoptosis in lung cancer. Therefore, it is possible that LACTB may suppress EMT by inhibiting Snail and increase the sensitivity of lung cancer cells to docetaxel. The above data suggest that LACTB is a good candidate for lung cancer therapy. However, further experiments are necessary before the clinical application of LACTB is realized.

In conclusion, LACTB showed a clinical correlation with the suppressed progression in lung cancer by negatively regulating cell migration, invasion and EMT while increasing apoptosis. LACTB exerts additive effects with docetaxel in the treatment of lung cancer cells. This study suggests that LACTB is a new suppressor for lung cancer.

\section{Acknowledgements}

Not applicable.

\section{Funding}

This research was supported by grants from the Natural Science Foundation of Shandong Province (ZR2019PC056), the Technology Development Program of Jinan City (201907117), and the Shandong Medical and Health Science and Technology Development Fund (202102080591).

\section{Availability of data and materials}

The datasets used and/or analyzed during the current study are available from the corresponding author on reasonable request.

\section{Authors' contributions}

HL and YW designed the study and reviewed the manuscript. YX acquired the majority of the data and drafted the manuscript. HS, MW and PH analyzed the data and revised the manuscript. MX and SH confirmed the authenticity of all raw data, interpreted the data, provided literature support and revised the manuscript. All authors read and approved the final manuscript for publication.

\section{Ethics approval and consent to participate}

Not applicable.

\section{Patient consent for publication}

Not applicable.

\section{Competing interests}

The authors declare that they have no competing interests.

\section{References}

1. Siegel RL, Miller KD, Fuchs HE and Jemal A: Cancer statistics, 2021. CA Cancer J Clin 71: 7-33, 2021.

2. Wu F, Wang L and Zhou C: Lung cancer in China: Current and prospect. Curr Opin Oncol 33: 40-46, 2021

3. Hirsch FR, Scagliotti GV, Mulshine JL, Kwon R, Curran WJ, Yi-Long W and Paz-Ares L: Lung cancer: current therapies and new targeted treatments. Lancet 389: 299-311, 2017.

4. de Sousa VM and Carvalho L: Heterogeneity in lung cancer. Pathobiology 85: 96-107, 2018.

5. Bai X, Meng L, Sun H,LiZ, Zhang X and Hua S: MicroRNA-196b inhibits cell growth and metastasis of lung cancer cells by targeting Runx2. Cell Physiol Biochem 43: 757-767, 2017.

6. Devlin JR and Verschuren EW: More than a tumor suppressor: E-Cadherin loss drives lung cancer metastasis. Am J Respir Cell Mol Biol 59: 141-142, 2018.

7. Ma Y, Wang L, He F, Yang J, Ding Y, Ge S, Fan X, Zhou Y, $\mathrm{Xu}$ X and Jia R: LACTB suppresses melanoma progression by attenuating PP1A and YAP interaction. Cancer Lett 506: 67-82, 2021.

8. Li HT, Dong DY, Liu Q, Xu YQ and Chen L: Overexpression of LACTB, a mitochondrial protein that inhibits proliferation and invasion in glioma cells. Oncol Res 27: 423-429, 2019.

9. Al-Ostoot FH, Salah S, Khamees HA and Khanum SA: Tumor angiogenesis: Current challenges and therapeutic opportunities. Cancer Treat Res Commun 28: 100422, 2021.

10. Zeng K, Chen X, Hu X, Liu X, Xu T, Sun H, Pan Y, He B and Wang S: LACTB, a novel epigenetic silenced tumor suppressor, inhibits colorectal cancer progression by attenuating MDM2-mediated p53 ubiquitination and degradation. Oncogene 37: 5534-5551, 2018. 
11. Peng LX, Wang MD, Xie P, Yang JP, Sun R, Zheng LS, Mei Y, Meng DF, Peng XS, Lang YH, et al: LACTB promotes metastasis of nasopharyngeal carcinoma via activation of ERBB3/EGFR-ERK signaling resulting in unfavorable patient survival. Cancer Lett 498:165-177, 2021.

12. Xie J, Peng Y, Chen X, Li Q, Jian B, Wen Z and Liu S: LACTB mRNA expression is increased in pancreatic adenocarcinoma and high expression indicates a poor prognosis. PLoS One 16 e0245908, 2021

13. Vasan N, Baselga J and Hyman DM: A view on drug resistance in cancer. Nature 575: 299-309, 2019.

14. Yang F, Yan Z, Nie W, Cheng X, Liu Z, Wang W, Shao C, Fu G and Yu Y: LACTB induced apoptosis of oxaliplatin-resistant gastric cancer through regulating autophagy-mediated mitochondrial apoptosis pathway. Am J Transl Res 13: 601-616, 2021.

15. Dibgre A and Weinberg RA: New insights into the mechanisms of epithelial-mesenchymal transition and implications for cancer. Nat Rev Mol Cell Biol 20: 69-84, 2019.

16. Jiang L, Huang J, Hu Y, Lu P, Luo Q and Wang L: Gli promotes tumor progression through regulating epithelial-mesenchymal transition in non-small-cell lung cancer. J Cardiothorac Surg 15: $18,2020$.

17. Xu W, Yu M, Qin J, Luo Y and Zhong M: LACTB regulates PIK3R3 to promote autophagy and inhibit EMT and proliferation through the $\mathrm{PI} 3 \mathrm{~K} / \mathrm{AKT} / \mathrm{mTOR}$ signaling pathway in colorectal cancer. Cancer Manag Res 12: 5181-5200, 2020.

18. Livak KJ and Schmitten TD: Analysis of relative gene expression data using real-time quantitative PCR and the 2(-Delta Delta C(T)) method. Methods 25: 402-408, 2001.

19. Tomczak K, Czerwińska $P$ and Wiznerowicz M: The cancer genome atlas (TCGA): An immeasurable source of knowledge. Contemp Oncol (Pozn) 19: A68-A77, 2015.

20. Heberle $E$ and Bardet AF: Sensitivity of transcription factors to DNA methylation. Essays Biochem 63: 727-741, 2019.

21. Xue C, He Y, Zhu W, Chen X, Yu Y, Hu Q, Chen J, Liu L, Ren F, Ren Z, et al: Low expression of LACTB promotes tumor progression and predicts poor prognosis in hepatocellular carcinoma. Am J Transl Res 10: 4152-4162, 2018.

22. Senga SS and Grose RP: Hallmarks of cancer-the new testament. Open Biol 11: 200358, 2021.
23. Mohammadian J, Sabzichi M, Molavi O, Shanehbandi D and Samadi N: Combined treatment with stattic and docetaxel alters the $\mathrm{Bax} / \mathrm{Bcl}-2$ gene expression ratio in human prostate cancer cells. Asian Pac J Cancer Prev 17: 5031-5035, 2016.

24. Zhang J, Wang J, Wong YK, Sun X, Chen Y, Wang L, Yang L, Lu L, Shen HM and Huang D: Docetaxel enhances lysosomal function through TFEB activation. Cell Death Dis 9: 614, 2018.

25. Colli LM, Machiela MJ, Zhang H, Myers TA, Jessop L, Delattre O, Yu K and Chanock SJ: Landscape of combination immunotherapy and targeted therapy to improve cancer management. Cancer Res 77: 3666-3671, 2017.

26. Asna N, Livoff A, Batash R, Debbi R, Schaffer P, Rivkind T and Schaffer M: Radiation therapy and immunotherapy-a potential combination in cancer treatment. Curr Oncol 25: e454-e460, 2018.

27. Pastushenko I and Blanpain C: EMT transition states during tumor progression and metastasis. Trends Cell Biol 29: 212-226, 2019.

28. Tulchinsky E, Demidov O, Kriajeska M, Barlev NA and Imyanitov E: EMT: A mechanism for escape from EGFR-targeted therapy in lung cancer. Biochim Biophys Acta Rev Cancer 1871: 29-39, 2019

29. Chae YK, Chang S, Ko T, Anker J, Agte S, Iams W, Choi WM, Lee K and Cruz M: Epithelial-mesenchymal transition (EMT) signature is inversely associated with T-cell infiltration in non-small cell lung cancer (NSCLC). Sci Rep 8: 2918, 2018.

30. Ribatti D, Tamma R and Annese T: Epithelial-mesenchymal transition in cancer: A historical overview. Transl Oncol 13: 100773, 2020.

31. Villarejo A, Cortés-Cabrera A, Molina-Ortiz P, Portillo F and Cano A: Differential role of Snaill and Snail2 zinc fingers in E-cadherin repression and epithelial to mesenchymal transition. J Biol Chem 289: 930-941, 2014.

32. Shen W, Pang H, Liu J, Zhou J, Zhang F, Liu L, Ma N, Zhang N, Zhang $\mathrm{H}$ and Liu L: Epithelial-mesenchymal transition contributes to docetaxel resistance in human non-small cell lung cancer. Oncol Res 22: 47-55, 2014.

This work is licensed under a Creative Commons Attribution-NonCommercial-NoDerivatives 4.0 International (CC BY-NC-ND 4.0) License. 